\title{
Desloratadine and depression, a drug safety signal based on worldwide spontaneous reporting of side effects
}

\author{
Jeltje Boer ${ }^{a *}$, Ellen Ederveen ${ }^{a *}$ and Birgitta Grundmark ${ }^{b, c}$

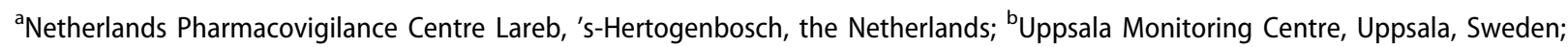 \\ 'Department of Surgery, Uppsala University, Uppsala, Sweden
}

\begin{abstract}
Objective: Desloratadine, a third-generation antihistamine, is claimed to cause fewer central nervous system (CNS) adverse drug reactions (ADRs) than antihistamines of the first- and second-generation. While literature is inconclusive regarding the possible CNS effects, symptoms like somnolence and hallucinations are acknowledged ADRs of desloratadine, indeed suggesting some passage of this drug across the blood-brain barrier. Depression is currently not described as an ADR in the approved desloratadine product labelling.

Materials and methods: In a joint signal detection workshop with the Uppsala Monitoring Centre and the Netherlands Pharmacovigilance Centre Lareb, case reports of suspected drug-ADR associations were analysed.

Results: Forty-nine unique case reports of desloratadine associated with depression or depressed mood were detected in the WHO global ADR database. In these reports, the median time to onset of depression was three days. Most patients recovered after withdrawal of desloratadine, and in five patients the symptoms of depression recurred after re-administration of desloratadine.

Conclusion: We hypothesize that desloratadine may enter the CNS and that it hence in rare cases may cause a clinically relevant state of depression, a relation that patients and their treating physicians should be made aware of.
\end{abstract}

\section{ARTICLE HISTORY}

Received 5 March 2018 Revised 8 May 2018 Accepted 13 June 2018

\section{KEY WORDS}

ADR; adverse drug reaction; antihistamine; depressed mood; depression; desloratadine; pharmacovigilance; safety signal; spontaneous reporting

\section{Background}

Desloratadine is a third-generation long-acting antihistamine which acts by competitively binding to the histamine 1 ( $\mathrm{H} 1)$-receptor $(1,2)$. It is an active metabolite of loratadine and is indicated for the symptomatic treatment of allergic rhinitis and urticaria (1). Antihistamines prevent the occurrence of histamine-activated processes (3). They inhibit smooth muscle contraction in bronchi and the gastrointestinal tract and inhibit the capillary permeability and the stimulation of sensory nerves. Thereby, they decrease allergic symptoms such as oedema, pruritus, and sneezing $(3,4)$.

H1-receptor antagonists, or antihistamines, are classified as first-, second-, and third-generation antihistamines. Firstgeneration, or classic antihistamines, are lipophilic, cross the blood-brain barrier easily, and hence have the propensity to cause central nervous system (CNS) side effects or adverse drug reactions (ADR), e.g. sedation. Second- and third-generation antihistamines, or new antihistamines, are more lipophobic and bind more selectively to the H1-receptor. Third-generation antihistamines are metabolites of the second-generation antihistamines and were developed with the intention to even further reduce CNS effects, although this has not been fully scientifically confirmed $(2,3)$. In high dosages they, to some extent, also cause sedation $(2,3,5)$.

Depression is a common, often chronic psychiatric disorder $(6,7)$. It is defined as a manifestation of depressed mood or loss of interest or pleasure which is present most of the day for at least two weeks, accompanied by at least four of the following symptoms: sleeping problems, low energy, change in appetite or weight, poor concentration or indecision, psychomotor retardation or agitation, thoughts of worthlessness or guilt, and suicidal thoughts (8). It affects functioning in daily life, quality of life, morbidity, and mortality $(7,9)$. Due to comorbidities, treatment costs, absence from work, and reduced productivity at work, depression leads to substantial costs for both patient and society (10).

Depression is neither acknowledged as an ADR in the European- nor the USA-approved product label for desloratadine $(1,11)$. The product label is the readily available and trusted source of information about drugs for prescribers and patients. The USA product label does mention emotional lability as an ADR in children 12-23 months old (11). Other CNS-ADRs mentioned in the European and USA product label are somnolence and hallucinations $(1,11)$. For loratadine, a second-generation antihistamine and a pro-drug of 
desloratadine, depression is mentioned in the Swiss labelling (12). In the USA product label for Claritin ${ }^{\circledR}$, the original loratadine brand, depression is reported to have occurred in at least one patient $(<2 \%)$ during the clinical trials (13).

In a bilateral workshop focused on detecting previously unknown side effects of drugs in a global database of ADR reports from patients and prescribers, a previously undescribed association between desloratadine and depression or depressed mood was detected. The aim of this article is to describe our hypothesis and raise awareness of this novel possible causal association.

\section{Materials and methods}

Systems to collect reports of ADRs spontaneously submitted by healthcare professionals and patients have been in place for decades in many high-income countries and are by now established in most countries globally. Such reporting systems are an effective means to detect rare safety signals that are unknown at the time of approval of drugs (14). A signal is in this context defined as a hypothesis of a risk with a medicine with data and arguments that support it. The reports are nationally collected for all drugs and primarily managed nationally at pharmacovigilance centres (and in parallel by marketing authorization holders of the drugs in question) which attempt to detect new signals from them. Once detected, the signals are conveyed to appropriate stakeholders (other drug authorities, marketing authorization holders, prescribers, and/or patients) to act upon as to constantly improve the safety knowledge and the wise use of approved drugs.

The WHO Collaborating Centre for International Drug Monitoring, the Uppsala Monitoring Centre (UMC), currently receives individual case safety reports (ICSRs) of suspected ADRs from 127 national pharmacovigilance centres around the world (15). The reports are stored in VigiBase, the WHO global database for ADRs which is used as a source for detecting, analysing, and communicating information on rare and previously unknown ADRs. VigiBase contains 16.0 million ICSRs (October 2017), and the detection of ADR signals in it is performed by means of advanced statistical methods.

The signal presented here of depression and depressed mood in relation to desloratadine was detected as one of several during a signal detection workshop focused on international patient ADR reporting performed by the UMC in collaboration with the Netherlands Pharmacovigilance Centre Lareb. Using statistical detection methods, drug-ADR combinations occurring disproportionately often in VigiBase were detected. A detailed description of the statistical signal detection algorithm used in the workshop has previously been described by Watson et al. (16). In brief, combinations were detected if the observed number of reports was significantly higher than what would be expected statistically had the reports been distributed randomly, and if the proportion of patient reports for the combination was high and the reports contained good-quality data, e.g. a narrative. Statistical signal detection methods are a means of

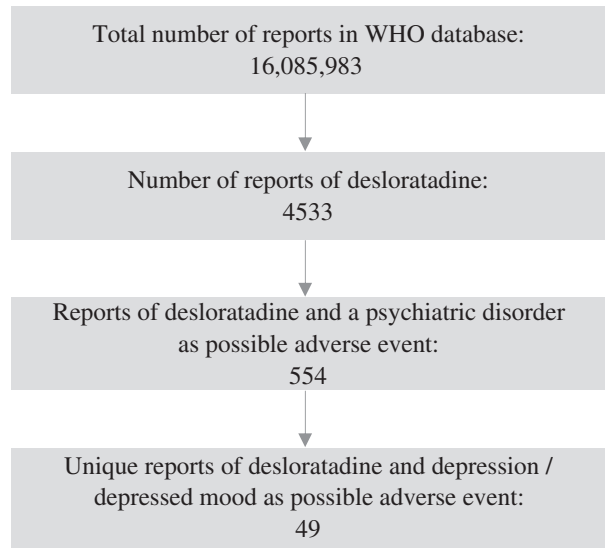

Figure 1. Number of reports in VigiBase on 28 October 2017 (15), structured following the Medical Dictionary for Regulatory Activities (MedDRA) (26).

prioritizing among reported combinations. Detected combinations hereafter need manual assessment to determine whether they contain information that would suggest a new causal relationship between drug and ADR. Current reference data for the drugs, the approved labelling in the EU and the USA, were scrutinized, and the narratives of the case series were systematically analysed. The now presented association of desloratadine and depression or depressed mood was one of the eight signals detected during the workshop that were deemed important to communicate to a wider audience (16).

\section{Results}

During the workshop the combination 'depressed mood' in relation to desloratadine was statistically detected for analysis as it contained 16 observed reports, whereas the expected number was 7. All reports of 'depression' in the database were considered clinically relevant to include into the case series for analysis. In October 2017, VigiBase hence contained 49 unique reports of patients experiencing a depressed mood or depression (from hereon in the case descriptions simplified as 'depression') associated with the use of desloratadine (Figure 1) (15). The reports originated from 11 different countries, of which the United States of America $(n=12)$, the Netherlands $(n=10)$, and Canada $(n=8)$ had reported the most. Eleven reports were serious according to established criteria, e.g. reactions causing fatality or hospitalization (17). Nearly half of the cases were reported by patients $(n=22)$. The reports concerned 28 women and 19 men (two reports lacked gender information) with an age range of 3-81 years (median 41 years).

In the 26 patients where symptom onset data were reported, the median time to onset was three days after start of desloratadine (ranging from 'several hours' after start to one year). One patient developed the reaction an uncertain period after the therapy with desloratadine was withdrawn. Besides depression, other psychiatric symptoms, e.g. hallucinations or anxiety, were co-reported in 18 of the cases. In six reports, thoughts of self-harm, suicidal ideation, or suicide attempt were co-reported. In children and adolescents $(n=5)$ co-reported symptoms included irritability, panic 


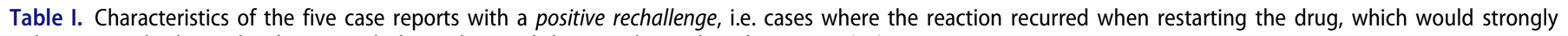
indicate causal relationship between desloratadine and depressed mood or depression (15).

\begin{tabular}{|c|c|c|c|c|c|c|c|}
\hline Case & Reporter & Age/Sex & $\begin{array}{l}\text { Suspected drugs; } \\
\text { daily dose; } \\
\text { indication }\end{array}$ & Concomitant drugs & Reactions reported & Time to onset & $\begin{array}{l}\text { Action with drug; } \\
\text { Outcome }\end{array}$ \\
\hline 1 & Patient & $42 / M$ & $\begin{array}{l}\text { Desloratadine; } \\
5 \text { mg } 2 \text { per day; } \\
\text { hay fever }\end{array}$ & - & Depressed mood & 12 hours & $\begin{array}{l}\text { Drug withdrawn; } \\
\text { recovered; recur- } \\
\text { rence of symptoms } \\
\text { on re-administration }\end{array}$ \\
\hline 2 & Patient & $38 / F$ & $\begin{array}{l}\text { Desloratadine; } \\
5 \text { mg per day; } \\
\text { hay fever }\end{array}$ & - & $\begin{array}{l}\text { Depressed mood, } \\
\text { anxiety, } \\
\text { dyspepsia }\end{array}$ & 5 days & $\begin{array}{l}\text { Drug withdrawn; } \\
\text { recovered; recur- } \\
\text { rence of symptoms } \\
\text { on re-administration }\end{array}$ \\
\hline 3 & Patient & $65 / F$ & $\begin{array}{l}\text { Desloratadine; } \\
\text { unknown; } \\
\text { urticaria }\end{array}$ & $\begin{array}{l}\text { Lansoprazole, doxepin, } \\
\text { bisoprolol, calcium, } \\
\text { hydroxyzine, } \\
\text { cholecalciferol, } \\
\text { hydrochlorothiazide }\end{array}$ & $\begin{array}{l}\text { Depression, } \\
\text { suicidal ideation }\end{array}$ & unknown & $\begin{array}{l}\text { Drug withdrawn; } \\
\text { recovered; recur- } \\
\text { rence of symptoms } \\
\text { on re-administration }\end{array}$ \\
\hline 4 & Patient & $16 / F$ & $\begin{array}{l}\text { Desloratadine; } \\
\text { unknown; } \\
\text { rhinitis allergic } \\
\text { n o s }\end{array}$ & - & $\begin{array}{l}\text { Depression, } \\
\text { suicidal ideation }\end{array}$ & unknown & $\begin{array}{l}\text { Drug withdrawn; reac- } \\
\text { tion abated; recur- } \\
\text { rence of symptoms } \\
\text { on re-administration }\end{array}$ \\
\hline 5 & Other & $48 / F$ & $\begin{array}{l}\text { Desloratadine; } \\
5 \text { mg unknown } \\
\text { times; unknown }\end{array}$ & Mometasone & $\begin{array}{l}\text { Depression, } \\
\text { thoughts of self- } \\
\text { harm, abnor- } \\
\text { mal crying }\end{array}$ & unknown & $\begin{array}{l}\text { Drug withdrawn; } \\
\text { recovered; recur- } \\
\text { rence of symptoms } \\
\text { on re-administration }\end{array}$ \\
\hline
\end{tabular}

reaction, hyperactivity, conduct disorder, and concentration impairment. Irritability was also co-reported by adults. There was no discernible difference in co-reported symptoms in elderly ( $\geq 60$ years; $n=6$ ) compared with adults aged between 18 and 60 years. Overall, at the time of reporting, 33 patients had recovered or were recovering from the depression. Of these, at least 30 had ceased using desloratadine. In the remaining three cases, information on treatment continuation or discontinuation was not reported. Depression recurred in five patients when the drug was restarted (Table I). In one patient, the depression did not recur after re-administration of desloratadine.

In some reports, other possible causes for the depression were described besides the use of desloratadine; in six patients, at least one other drug (montelukast, xylometazoline, loratadine, permethrin, diphenhydramine, tolterodine, levothyroxine, triamcinolone, paroxetine) was co-reported as suspected to have caused the depression. Depression is an acknowledged ADR for three of these: montelukast, loratadine, and triamcinolone $(12,13,18,19)$. For paroxetine, suicidal ideation and suicidal behaviour are described ADRs, although these symptoms may also be due to an underlying disease (20). Other concomitant medications for which depression or related symptoms are acknowledged potential ADRs were used by 12 patients but were not suspected by the reporter to have caused the events. Another five patients used an antidepressant drug, and one patient had a history of depression. Two reports describe suspected interactions between desloratadine and pre-existing use of fluoxetine and citalopram, respectively. To date, no such interactions are known in literature (21). The starting date and other details surrounding the treatment with most of the concomitant medications were unknown. Two patients had hypothyroidism in their medical history, and drug dependence was reported as possible ADR of desloratadine in one case alongside four other co-reported psychiatric events, without further details given. Data on other risk factors, such as family history or social influences, were not reported.

\section{Discussion}

Depression is not included as an acknowledged ADR of desloratadine in European- or USA-approved product labels, while other psychiatric and CNS symptoms, like hallucinations and somnolence, are $(1,11)$. Depression being a severe disorder negatively affecting quality of life, medical morbidity, and life expectancy (9) renders our hypothesis of it being a potential side effect of desloratadine important to be publicly known.

In October 2017, VigiBase contained 49 spontaneous reports of depression or depressed mood suspected to be caused by desloratadine treatment. The age-specific clinical presentation of depression, where paediatric patients may present with feelings of guilt, irritability, and disturbances in school functioning (22), is in accordance with the findings in our case series and hence supports the signal. The median time to onset of three days in the case series is plausible for a direct pharmacological effect, and although possible confounders were present in some reports 30 patients recovered from depression after withdrawal of desloratadine. The perhaps strongest argument for a causal relationship between desloratadine and depression is the five reports of patients in whom the symptoms of depression recurred when desloratadine was re-administered. Unfortunately, in three of these reports, information about the time to onset is missing.

Data from spontaneous reporting from health care professionals and patients have limitations. In individual reports the certainty of causality between the reported drug and the subsequent suspected reaction is variable, as is the level of detail of clinical data provided. With its inherent biases and underreporting, spontaneous reporting does not allow for true incidences of suspected ADRs to be calculated. The 
method is, however, a powerful tool to detect hypotheses of safety issues or signals, for further analysis using other methods.

The USA product label describes that in clinical trials $3.1 \%$ of the children between 12 and 23 months of age developed emotional lability after receiving desloratadine, compared with none in the placebo group (11). The side effects currently included in the labelling for desloratadine suggest that desloratadine may cross the blood-brain barrier, contrary to what is communicated elsewhere in the labelling $(1,11)$. Interestingly, for loratadine, the pro-drug of desloratadine, depression is labelled in at least one nationally approved drug label (12).

The reports in VigiBase are supported by sources that do not exclude that desloratadine may enter the CNS in humans $(1,11,12)$. Results of studies on CNS effects of desloratadine are somewhat inconsistent. The USA product label includes information that in preclinical animal studies desloratadine does 'not readily cross the blood-brain barrier' (11), indicating that passage to some extent is not entirely excluded. In 2009 , Layton et al. presented the safety of desloratadine in a study of 11,828 patients with a median age of 37 years; this was done by retrospectively collecting data from general practitioner prescribers via a questionnaire. Depression was reported in a small group of patients $(n=16)$ during their first month of desloratadine treatment. Malaise and lassitude were in the study, however, the only psychiatric events assessed as possibly related to desloratadine (23). In 2013, González-Núñez et al. reviewed published data on the safety profile of desloratadine and concluded that it has no CNS effects and does not cross the blood-brain barrier (24). This conclusion differs, as noted above, somewhat from what is found in the USA labelling. These apparent discrepancies might be explained by the fact that labelling approved by a regulatory authority may be based partly also on unpublished data made available to them by the marketing authorization holder. In a 2014 study of 92 patients treated for chronic pruritus, Ozdemir et al. compared the effects on mood between treatment with classic and new-generation antihistamines. Using 'the profile of mood states' questionnaire, no significant difference in the influence on mood was seen between the treatment groups. Regarding the individual antihistamines the authors then make the somewhat conflicting claim that cetirizine and hydroxyzine had a more negative influence on mood than desloratadine, rupatadine, and levocetirizine (25), which again seems to indicate at least some influence by the latter three substances on patient mood. Overall, available sources suggest that desloratadine may cross the blood-brain barrier to some extent in humans.

Our opinion is that the collected nature of the spontaneously reported cases from reporters worldwide in the VigiBase case series, in conjunction with other available scientific data, suggests that the widely used anti-allergic drug desloratadine in rare cases may be causally related to occurrence of depression. The suicidal ideation and suicide attempt co-reported in some of the reports reinforce that the severity of desloratadine-induced depression may be profound; patients and healthcare professionals should be aware of this.

\section{Acknowledgements}

The authors are indebted to the national centres that make up the WHO Programme for International Drug Monitoring and contribute reports to VigiBase. The opinions and conclusions of this study are, however, not necessarily those of the various centres nor of the WHO. The authors would also like to thank Florence van Hunsel and Linda Härmark at Lareb for their valuable input to the article.

\section{Disclosure statement}

No potential conflict of interest was reported by the authors.

\section{Funding}

No financial support was received for the conduct of this study or preparation of this manuscript.

\section{Notes on contributors}

Jeltje Boer, MD, is scientific assessor at the Netherlands Pharmacovigilance Centre Lareb, 's-Hertogenbosch, the Netherlands.

Ellen Ederveen, MD, is scientific assessor at the Netherlands Pharmacovigilance Centre Lareb, 's-Hertogenbosch, the Netherlands.

Birgitta Grundmark, MD, PhD, is senior researcher at Uppsala Monitoring Centre and researcher at the Department of Surgery at Uppsala University, Uppsala, Sweden.

\section{ORCID}

Birgitta Grundmark (iD http://orcid.org/0000-0002-2799-9374

\section{References}

1. Summary of products characteristics for desloratadine (Azomyr ${ }^{\circledR}$ ) [Internet]. European Medicines Agency; 2006 [cited 2017 Jun 28]. Available at: http://www.ema.europa.eu/docs/en_GB/document library/EPAR_-_Product_Information/human/000310/WC500030164. pdf

2. Verster JC, Volkerts ER. Antihistamines and driving ability: evidence from on-the-road driving studies during normal traffic. Ann Allergy Asthma Immunol. 2004;92:294-303.

3. Camelo-Nunes IC. New antihistamines: a critical view. J Pediatr (Rio J). 2006;82:173-80.

4. Brunton LL, Lazo JS, Parker KL. Goodman \& Gilman's The pharmacological basis of therapeutics. 11th ed. New York (NY): McGrawHill; 2006.

5. Simons FE, Simons KJ. Clinical pharmacology of new histamine $\mathrm{H}_{1}$ receptor antagonists. Clin Pharmacokinet. 1999;36:329-52.

6. Thornicroft G, Chatterji S, Evans-Lacko S, Gruber M, Sampson N, Aguilar-Gaxiola $S$, et al. Undertreatment of people with major depressive disorder in 21 countries. Br J Psychiatry. 2017;210: 119-24.

7. Bohman $H$, Jonsson $U$, Päären $A$, Von Knorring $A L$, Olsson $G$, Von Knorring L. Long-term follow-up of adolescent depression. Ups J Med Sci. 2010;115:21-9.

8. American Psychiatric Association. Diagnostic and statistical manual of mental disorders, fourth edition (DSM-4). Koster van Groos GAS translator. Amsterdam: Harcourt Assessment BV; 2000. p. 210-12. 
9. Kessler RC, Bromet EJ. The epidemiology of depression across cultures. Annu Rev Public Health. 2013;34:119-38.

10. Greenberg PE, Fournier AA, Sisitsky T, Pike CT, Kessler RC. The economic burden of adults with major depressive disorder in the United States (2005 and 2010). J Clin Psychiatry. 2015;76:155-62.

11. Product label for desloratadine (Clarinex ${ }^{\circledR}$ ) [Internet]. US Food and Drug Administration; 2014 [cited 2017 Jun 28]. Available at: https://www.accessdata.fda.gov/drugsatfda_docs/label/2014/ 021165s017,021300s014,021312s015,021563s003lbl.pdf

12. Summary of products characteristics for loratadine (Claritine ${ }^{\circledR}$ ) [Internet]. Swissmedic Schweizerisches Heilmittelinstitut; 2017 Jan [cited 2017 Sep 19]. Available at: http://www.swissmedicinfo.ch/ default.aspx

13. Product label for loratadine $\left(\right.$ Claritin $\left.^{\circledR}\right)$ [Internet]. US Food and Drug Administration; 2000 [cited 2017 Aug 23]. Available at: https://www.accessdata.fda.gov/drugsatfda_docs/label/2000/ 20641s7lbl.pdf

14. van Hunsel F, Härmark L, Pal S, Olsson S, van Grootheest K. Experiences with adverse drug reaction reporting by patients: an 11-country survey. Drug Saf. 2012;35:45-60.

15. VigiBase [Internet]. WHO Global Individual Case Safety Reports database. Uppsala: Uppsala Monitoring Centre; 2017 [cited 2017 Sep 01]. Available at: https://www.who-umc.org/ [access restricted].

16. Watson S, Chandler RE, Taavola H, Härmark L, Grundmark B, Zekarias $A$, et al. Safety concerns reported by patients identified in a collaborative signal detection workshop using VigiBase: results and reflections from Lareb and Uppsala Monitoring Centre. Drug Saf. 2018;41:203-12.

17. Guideline on good pharmacovigilance practices (GVP). Module VI Management and reporting of adverse reactions to medicinal product [Internet]. European Medicines Agency; 2014 Sep [cited 2017
Sep 14]. Available at: http://www.ema.europa.eu/docs/en_GB/document_library/Scientific_guideline/2014/09/WC500172402.pdf

18. Summary of product characteristics for montelukast [Internet]. Electronic Medicines Compendium; 2017 Jan [cited 2017 Nov 10]. Available at: http://www.medicines.org.uk/emc/medicine/28220

19. Summary of product characteristics for triamcinolone [Internet]. Electronic Medicines Compendium; 2017 Oct [cited 2017 Nov 12] Available at: http://www.medicines.org.uk/emc/medicine/28913

20. Summary of product characteristics for paroxetine [Internet]. Electronic Medicines Compendium; 2016 Mar [cited 2017 Nov 12]. Available at: http://www.medicines.org.uk/emc/medicine/26965

21. Baxter K, editor. Stockley's Drug Interactions [Internet]. London: Pharmaceutical Press; 2017 [cited 2017 Aug 05]. Available at: http://www.medicinescomplete.com [access restricted].

22. Bonin L. Pediatric unipolar depression: epidemiology, clinical features, assessment, and diagnosis [Internet]. Waltham (MA): UpToDate; 2017 [cited 2017 Nov 12]. Available at: https://www. uptodate.com/contents/pediatric-unipolar-depression-epidemiolo gy-clinical-features-assessment-and-diagnosis [access restricted].

23. Layton D, Wilton L, Shakir SA. Examining the tolerability of the non-sedating antihistamine desloratadine: a prescription-event monitoring study in England. Drug Saf. 2009;32:169-79.

24. González-Núñez V, Valero A, Mullol J. Safety evaluation of desloratadine in allergic rhinitis. Drug Saf. 2013;12:445-53.

25. Ozdemir PG, Karadag AS, Selvi Y, Boysan M, Bilgili SG, Aydin A, et al. Assessment of the effects of antihistamine drugs on mood, sleep quality, sleepiness, and dream anxiety. Int J Psychiatry Clin Pract. 2014;18:161-8.

26. Medical Dictionary for Regulatory Activities (MedDRA) [Internet]. International Council for Harmonisation of Technical Requirements for Pharmaceuticals for Human Use. [cited 2018 Apr 27]. Available at: https://www.meddra.org/ 